\title{
Introduction to the D-SPECT for Technologists: Workflow Using a Dedicated Digital Cardiac Camera
}

\author{
Robert D. Johnson ${ }^{1}$, Navkanwal Kaur Bath ${ }^{1}$, Jeffrey Rinker ${ }^{1}$, Stephen Fong ${ }^{1}$, Sara St. James ${ }^{2}$, Miguel Hernandez \\ Pampaloni $^{3}$, and Thomas A. Hope ${ }^{1,3}$

\begin{abstract}
${ }^{I}$ Department of Radiology, San Francisco VA Medical Center, San Francisco, California; ${ }^{2}$ Department of Radiation Oncology, University of California, San Francisco, San Francisco, California; and ${ }^{3}$ Department of Radiology and Biomedical Imaging, University of California, San Francisco, San Francisco, California
\end{abstract}

CE credit: For CE credit, you can access the test for this article, as well as additional JNMT CE tests, online at https://www.snmmilearningcenter.org.
Complete the test online no later than December 2023. Your online test will be scored immediately. You may make 3 attempts to pass the test and must
answer $80 \%$ of the questions correctly to receive $1.0 \mathrm{CEH}$ (Continuing Education Hour) credit. SNMMI members will have their CEH credit added to their VOICE
transcript automatically; nonmembers will be able to print out a CE certificate upon successfully completing the test. The online test is free to SNMMI members; nonmembers must pay $\$ 15.00$ by credit card when logging onto the website to take the test.

The D-SPECT is a dedicated cardiac camera that incorporates a solid-state semiconductor detector. This camera differs greatly from conventional SPECT/CT systems, resulting in significant differences in patient imaging. This continuing education article focuses on the specifications of both SPECT/CT and D-SPECT systems, radiopharmaceutical dosing requirements, imaging workflows, and some disadvantages of using each camera system. When used properly, the D-SPECT system can provide high-quality cardiac images with lower doses and faster exam times than conventional SPECT/CT systems.

Key Words: CNMT; SPECT; cardiac; D-SPECT; digital; SPECT

J Nucl Med Technol 2020; 48:297-303

DOI: $10.2967 /$ jnmt.120.254870

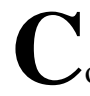

oronary artery disease is the leading cause of death in the United States (1). Myocardial perfusion SPECT is one of the most frequently used diagnostic tools for patients at risk for coronary artery disease and has significant prognostic value (2). Dedicated cardiac SPECT cameras such as the D-SPECT (Spectrum Dynamics) have been designed specifically for myocardial perfusion imaging studies. Equipped with cadmium-zinc-telluride (CZT)-based detectors, the D-SPECT has demonstrated image quality superior to that of standard SPECT or SPECT/CT while reducing patient and personnel radiation exposure and decreasing imaging time (3).

Received Aug. 5, 2020; revision accepted Sep. 23, 2020.

For correspondence or reprints contact: Thomas A. Hope, University of California, San Francisco, 505 Parnassus Ave., San Francisco, CA 94143.

E-mail: thomas.hope@ucsf.edu

Published online Oct. 5, 2020.

COPYRIGHT (c) 2020 by the Society of Nuclear Medicine and Molecular Imaging.

\section{CONVENTIONAL SPECT/CT}

SPECT imaging is based on 50-y-old technology that consists of a collimator and an NaI crystal coupled to photomultiplier tubes. The $\gamma$-rays emitted from the patient after radiotracer injection pass through the collimator and interact with the crystal, resulting in thousands of optical scintillation photons, which are detected by the photomultiplier tubes. The role of the photomultiplier tubes is to convert the optical signal from the scintillation photons into an electronic signal proportional to the energy deposited in the crystal. The thickness of the NaI crystal typically ranges from 6.35 to $15.9 \mathrm{~mm}$ (4). For ${ }^{99 \mathrm{~m}} \mathrm{Tc}$-labeled radiotracers, a thickness of $9.5 \mathrm{~mm}$ is preferred as it is able to stop $90 \%$ of the 140-keV photons incident on the crystal (4).

Most SPECT/CT cameras used for cardiac imaging have 2 detectors configured at $90^{\circ}$ or $180^{\circ}$ mounted on a gantry, although 3-detector systems are also used. In the practice of nuclear cardiac imaging, the preferred configuration of the dual-detector system is $90^{\circ}$, as this increases the sensitivity of images and reduces the scanning time (4). Since the heart is positioned anteriorly in the left hemithorax, supine imaging begins at a $45^{\circ}$ right anterior oblique angle and completes a $180^{\circ}$ arc around the patient.

The gantry rotates while the detectors contour around the patient's chest as part of the setup process for image acquisition, and 64 images with 32 steps are typically acquired. Conventional SPECT cameras are equipped with low-energy, high-resolution parallel-hole collimators; the role of the parallel-hole collimators is to limit the detected photons to those perpendicular to the detector, making tomographic image reconstruction possible. These same collimators, however, will stop more than $99.9 \%$ of photons because they are not incident on the holes of the collimator, resulting in a tradeoff between spatial resolution and sensitivity. Because of this loss of sensitivity, acquisition times on the order of $20 \mathrm{~min}$ are required for SPECT studies (4). 


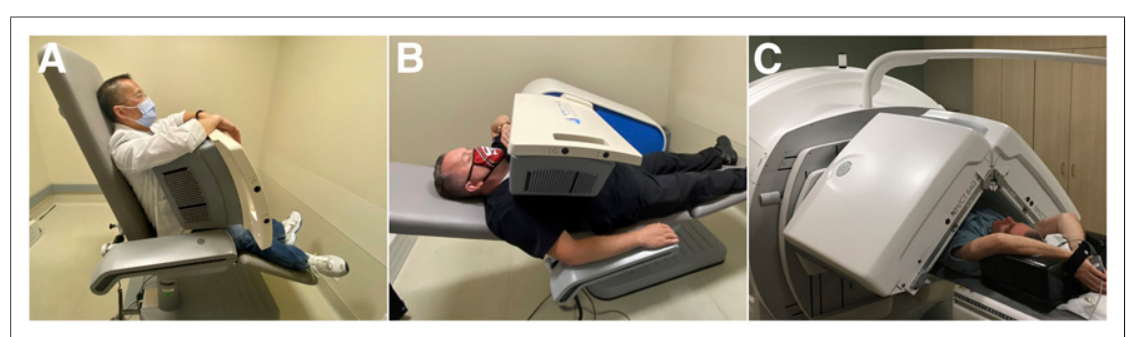

FIGURE 1. Multiple imaging positions can help to compensate for lack of attenuation correction. (A) Upright positioning on D-SPECT at approximately $70^{\circ}$ chair angle, with detector at its closest position to patient. Patient can rest both arms on top of detector at about eye level, or right arm may be placed on arm rest. (B) Supine positioning on D-SPECT at approximately $30^{\circ}$ chair angle. Right arm may be placed on arm rest, with left arm on top of camera or over patient's head. (C) Supine positioning on conventional SPECT camera, with both detectors at $90^{\circ}$ and arms over head.

high-resolution collimators, which typically have holes measuring $35-45 \times$ $1.5-2.0 \mathrm{~mm}$. Because of the increased aperture size relative to the size of the collimator, there is an 8-fold increase in acceptance of incidental photon detection, compared with conventional SPECT collimators $(6)$. When the gantry is stationary and the detector columns rotate individually, the D-SPECT enables a list mode for dynamic acquisition. Because of geometry and sensitivity limitations, conventional SPECT systems typically cannot acquire dynamic datasets (6).

One unique aspect of the D-SPECT

The hybrid technology of SPECT/CT is used for myocardial imaging, with the CT component applied in tandem with the SPECT component. Because all SPECT/CT studies are acquired sequentially, it is important that the patient be in the exact same position during and between each scan (apart from normal tidal breathing). Undesirable movement by the patient will cause a misregistration of the SPECT and CT images. In addition to anatomic information, the CT component of SPECT/CT systems is used to correct for attenuation (Fig. 1) (5).

\section{D-SPECT EQUIPMENT}

Unlike the original SPECT technology, which relied on the pairing of scintillators to photomultiplier tubes, the DSPECT detector uses semiconductor-based solid-state detectors that are constructed with CZT. When the photons interact with the CZT in the semiconductor, the incident $\gamma$-rays create electron pairs and an electrical signal is produced. The direct conversion of energy in the semiconductor solid-state detectors is characterized by good energy resolution, and in clinical applications the energy resolution is reported to be better than $6 \%$ at $140 \mathrm{keV}$ (5). Unlike other detectors (e.g., germanium), CZT operates at room temperature.

Though there are several types of CZT camera, this review focuses on the D-SPECT (Spectrum Dynamics). In the D-SPECT, there are 9 individually rotating detector columns arranged on a stationary gantry in a curved configuration to accommodate the heart on the left side of the patient's chest (Fig. 2). Each detector column is $40 \mathrm{~mm}$ wide (16 pixels) by $160 \mathrm{~mm}$ tall (64 pixels). The columns comprise CZT detectors aligned with the square apertures of the parallel-hole tungsten collimator in front of them, with 1 CZT pixel for each square aperture. Every detector column rotates at $110^{\circ}$ independently of the other columns, allowing acquisition of hundreds of projections at varying angles of the heart. The tungsten collimator has short holes $(21.7 \mathrm{~mm})$, and the square apertures of the collimator $(2.26 \mathrm{~mm})$ allow a higher percentage of photons to pass through than is possible with conventional low-energy, system is that patients are imaged while sitting and can be positioned upright or supine (Fig. 3). An additional and important difference between the D-SPECT and conventional SPECT/CT systems is the absence of CT imaging for attenuation correction. Approaches to minimize attenuation artifacts are discussed below.

\section{COMPARISON OF IMAGE QUALITY BETWEEN SYSTEMS}

In nuclear medicine, a $\gamma$-camera is used to produce an image, and the quality of these images depends on 3 factors: energy resolution, spatial resolution, and sensitivity.

Energy resolution characterizes the ability of the system to determine the energy of the incident photons. In nuclear medicine, good energy resolution allows for the discrimination of low-energy photons (that have already scattered in the patient) from higher-energy photons (that have had fewer interactions before depositing their energy in the detector). Energy resolution is reported as the full width at half maximum of a gaussian fit of the energy profile, for a given energy. A gaussian function is a function that represents the normal distribution, and the full width at half maximum is the energy profile width at which the measured

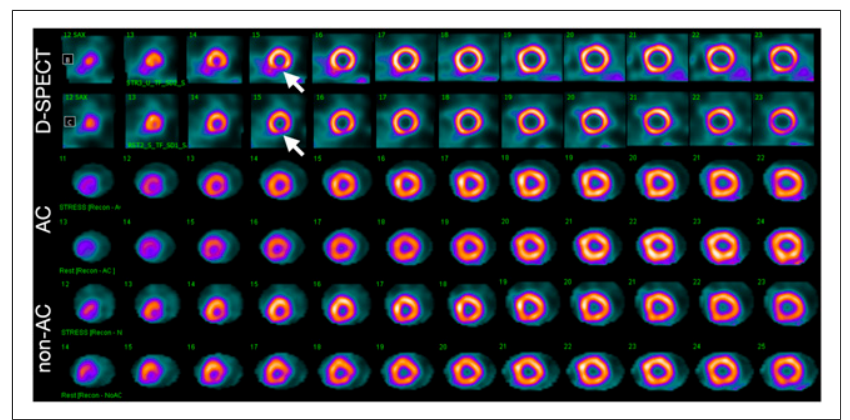

FIGURE 2. Example of distal inferior wall attenuation artifact (decreased uptake, arrows) seen occasionally on both rest and stress D-SPECT images. Myocardium is delineated more sharply with D-SPECT than with attenuation-corrected $(A C)$ or non-AC SPECT/CT. 


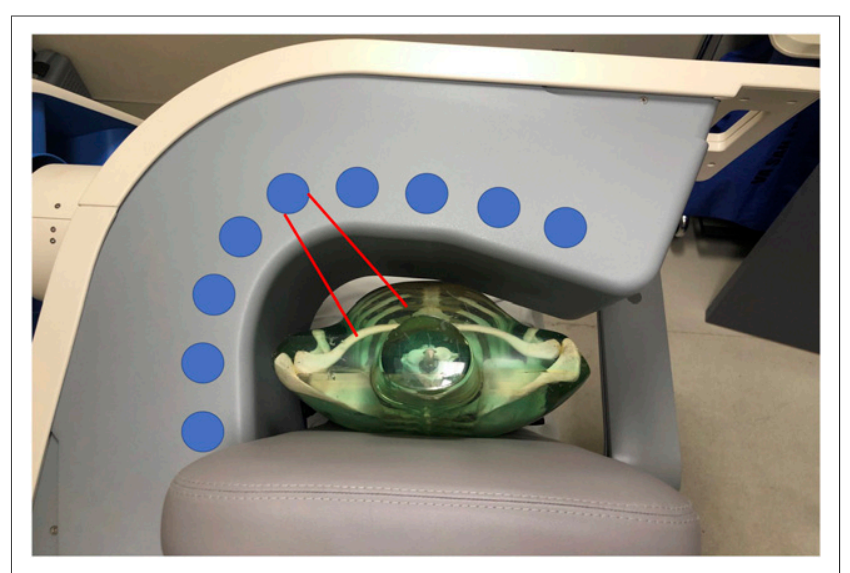

FIGURE 3. Aerial view of camera heads arranged inside gantry, showing approximate angles taken to achieve images. Red lines indicate heart-centric FOV of 1 of 9 detectors.

energy is half its maximum value. Because energy resolution depends on the incident photon energy and will change depending on the radionuclide being imaged, the energy of the measurement is always reported. CZT has better energy resolution than $\mathrm{NaI}$ across the clinically relevant energy range, with the energy resolution being improved by a factor of 2 or better (7).

Spatial resolution is the ability to separate distinct objects, with better resolution resulting in sharper, more detailed images. The spatial resolution of the D-SPECT system is $2.5 \mathrm{~mm}$, whereas traditional SPECT systems typically have a spatial resolution of 3.5-4.0 mm (4) (Table 1) because of the geometry of the system and the small size of the individual detector elements.

Sensitivity is reported as the percentage of emitted photons from the patient that are detected by the SPECT system. In a system with higher sensitivity, an image acquired during the same length of time as on a lowersensitivity system will have more counts and a better signalto-noise ratio. In the D-SPECT module, each detector column turns individually on its own axis, and a greater number of photons pass through the larger-hole collimators. These features increase the sensitivity of the D-SPECT by $5-10$ times over the traditional SPECT system (4). For conventional SPECT systems, sensitivity is rather low because only a small

TABLE 1

Characteristics of Crystals Used in Cardiac SPECT and D-SPECT Detectors (4)

\begin{tabular}{lcccc}
\hline $\begin{array}{c}\text { Detector } \\
\text { crystal }\end{array}$ & $\begin{array}{c}\text { Thickness } \\
(\mathrm{mm})\end{array}$ & $\begin{array}{c}\text { Energy } \\
\text { resolution } \\
(\%)\end{array}$ & $\begin{array}{c}\text { Intrinsic } \\
\text { spatial } \\
\text { resolution } \\
(\mathrm{mm})\end{array}$ & $\begin{array}{c}\text { Count } \\
\text { rate } \\
(\mathrm{kcps})\end{array}$ \\
\hline $\mathrm{Nal}$ & 9.5 & 10 & 4 & $\geq 250$ \\
$\mathrm{CZT}$ & 5 & 6 & 2.5 & $\geq 600$ \\
\hline
\end{tabular}

fraction $\left(10^{-6}\right)$ of photons are detected from the radiotracer activity because of absorption by the collimator (3) and the solid-angle coverage.

Image quality on conventional SPECT cameras can be impacted by various artifacts. These are caused by a relatively low number of photons emitted from the heart that are overwhelmed by activity from regions such as the intestines or biliary system. This situation can result in the ramp filter artifact, in which activity below the diaphragm results in either higher or lower activity in the inferior wall $(4,8)$. Direct alignment with the heart, combined with D-SPECT's approximately $15.7-\mathrm{cm}$ axial field of view (FOV) and its concentrated heart-centric aiming, decreases the number of acquired photons from extracardiac areas, minimizing this artifact. One risk with the smaller FOV of the D-SPECT is that truncation artifact can occur if the heart is not within the FOV of the scanner (4).

\section{DOSING INFORMATION}

Studies show that the D-SPECT allows injection of a lower administered activity to reduce radiation exposure while providing diagnostic images within a shorter acquisition time (Table 2). The guidelines of the American Society of Nuclear Cardiology recommend 296-444 MBq (8-12 mCi) for rest imaging and $888-1,332 \mathrm{MBq}(24-36 \mathrm{mCi})$ for stress imaging in a 1-d rest-stress protocol (9). In an article by Perrin et al., this dose was reduced to $111-259 \mathrm{MBq}$ (3-7 $\mathrm{mCi})$ and $370-814 \mathrm{MBq}(10-22 \mathrm{mCi})$, respectively, on the DSPECT (10), although this study used a stress-rest protocol. In the Perrin article, this dose range resulted in an acquisition time of $7.7 \mathrm{~min}$ at stress and $2.7 \mathrm{~min}$ at rest. Use of the DSPECT would reduce the effective dose from roughly $13 \mathrm{mSv}$ using a standard SPECT camera to $8 \mathrm{mSv}$. Radiation exposure can be reduced further, particularly in obese patients, by using upright positioning, which is helpful for interpretation, and use of stress-first imaging can eliminate the need for rest scans (11). Using similar low administered activities, multiple studies have shown D-SPECT imaging to have a high accuracy compared with invasive angiography $(12,13)$. Overall, the ability to administer an overall lower activity reduces exposure not only to patients but also to the technologist performing the studies.

\section{D-SPECT WORKFLOW}

\section{Preparation}

Patient preparation for cardiac imaging with the DSPECT is the same as for conventional SPECT/CT, including the need to refrain from caffeinated beverages and to fast for at least $4 \mathrm{~h}$ beforehand. Limitations on other cardiac and systemic medications are not different from standard myocardial perfusion imaging protocols. Providing water after the rest injection can help minimize uptake in the liver, bowel, and gallbladder, as can longer delays between injection and imaging, and both are common methods for decreasing artifacts. 
TABLE 2

Same-Day Protocols with D-SPECT Using 99mTc-Based Radiotracer

\begin{tabular}{cll}
\hline \multicolumn{1}{c}{ Protocol } & \multicolumn{1}{c}{ Rest study } & \multicolumn{1}{c}{ Stress study } \\
\hline Rest/stress $(4,9)$ & $296-444 \mathrm{MBq}(8-12 \mathrm{mCi})$ (conventional SPECT) & 888-1,332 MBq (24-36 mCi) (conventional SPECT) \\
Rest/stress (12) & $185 \mathrm{MBq}(5.0 \mathrm{mCi})$ for $<91 \mathrm{~kg}$ & $463 \mathrm{MBq}(12.5 \mathrm{mCi})$ for $<91 \mathrm{~kg}$ \\
& $370 \mathrm{MBq}(10.0 \mathrm{mCi})$ for $>91 \mathrm{~kg}$ & $925-1,110 \mathrm{MBq}(25.0-30.0 \mathrm{mCi})$ for $>91 \mathrm{~kg}$ \\
Stress/rest (10) & $359 \mathrm{MBq}(9.7 \mathrm{mCi})$ for $75 \mathrm{~kg}$ & $118 \mathrm{MBq}(3.2 \mathrm{mCi})$ for $75 \mathrm{~kg}$ \\
& $814 \mathrm{MBq}(22 \mathrm{mCi})$ for $>110 \mathrm{~kg}$ & $259 \mathrm{MBq}(7.0 \mathrm{mCi})$ for $>110 \mathrm{~kg}$ \\
Stress/rest (11) & $648-1,147 \mathrm{MBq}(17.5-31.0 \mathrm{mCi})$ based on BMl & $207-929 \mathrm{MBq}(5.6-25.1 \mathrm{mCi})$ based on BMl
\end{tabular}

Doses are based on weight and body mass index (BMI).

Recommended doses from ASNC guidelines $(4,9)$ are weight-based and about $50 \%$ greater than doses from the other guidelines (10-12).

\section{Positioning}

The curved configuration of the D-SPECT allows for the heart to be imaged with the camera head at the closest possible position to the patient. During the acquisition, the patient may either sit upright $\left(70^{\circ}\right)$ or recline supine $\left(30^{\circ}\right)$ with the arms placed on the detector (Fig. 3). The seat angle may be adjusted to any degree within the range, to help compensate for subdiaphragmatic artifacts. Variations in body habitus are accommodated by the adjustable seat. Once seated, the patient is positioned as far to the left as possible (slightly off center) while maintaining an erect and parallel position in the chair (no slouching if possible). Moving the chair is also necessary to position the heart in the FOV. The technologist, using the push-button hand controller, manually aligns the camera head to closely fit against the anterior chest and left lateral thoracic cavity (Fig. 2). Overall, the ability to image upright patients on SPECT equipment is more comfortable for them than is supine imaging.

Upright positioning is especially helpful when imaging patients with chronic obstructive pulmonary disease or dyspnea, as lying flat often exacerbates shortness of breath. The option of imaging upright can make the difference in whether a stress test exam can be completed (14). Frequently, the patient's arms are placed overhead on a conventional SPECT/CT device, but the D-SPECT requires that only the left arm be partially elevated. Regardless of the chair angle, the patient may choose either to rest both arms directly on the camera itself or to rest the right arm on the movable armrest.

Conventional SPECT imaging may require a patient to be prone to minimize artifacts. With the D-SPECT, an alternative is to adjust the chair angle (Fig. 3), which eliminates the discomfort associated with prone imaging (12), and these various positions can reduce artifacts $(3,15)$. One study demonstrated the benefit of patient positioning with the D-SPECT in obese patients by comparing upright and supine views from the D-SPECT with a supine view from a conventional SPECT camera (11). Of the 101 patients on the SPECT camera, 74 needed a rest scan, whereas only 42 of the 101 patients scanned in an upright position on the DSPECT required a rest scan. The study concluded that the addition of an upright image in obese patients improved the ability to determine whether rest imaging was necessary, thus reducing the radiation exposure to the patients (11).

\section{Detector Alignment}

When setting up for imaging with the D-SPECT, the technologist has to approximate the heart alignment until the appropriate steps are taken on the acquisition computer during the prescan. Because of the D-SPECT's smaller FOV, localizing the heart can be difficult at first. Unlike conventional imaging using a live persistence-scope, D-SPECT data are initially collected on the acquisition computer as a prescan. The alignment process takes a few seconds and aids in ensuring that the myocardium is centered within the FOV. This process may be repeated as many times as necessary to align the heart within 3 different regions of interest: anterior, lateral, and axial (aerial). The technologist centers the heart in all 3 planes displayed during the prescan to ensure the heart is centered within the FOV (Fig. 4) (16).

This system of aligning the heart is unique to D-SPECT and may require some trial and error by the technologist. The initial alignment and prescan are especially important because processing with the D-SPECT requires an accurate prescan, which is not needed on a conventional SPECT/CT or SPECT-only system. The time spent in prescanning saves time during postprocessing and limits the need for reimaging. Once the heart is located and regions of interest are drawn, image acquisition can begin.

\section{Imaging}

Once imaging is under way, neither the patient nor the camera moves. The only time the camera heads are moving is during the setup process, when the technologist manually moves the detector. This reduces the possibility of motioncreated artifacts or injuries. Lack of motion and noise, combined with the open seating configuration, decreases concerns of claustrophobia. The curved configuration of the D-SPECT, as it touches the patient just below the axilla, does not interfere with the patient's line of sight. 


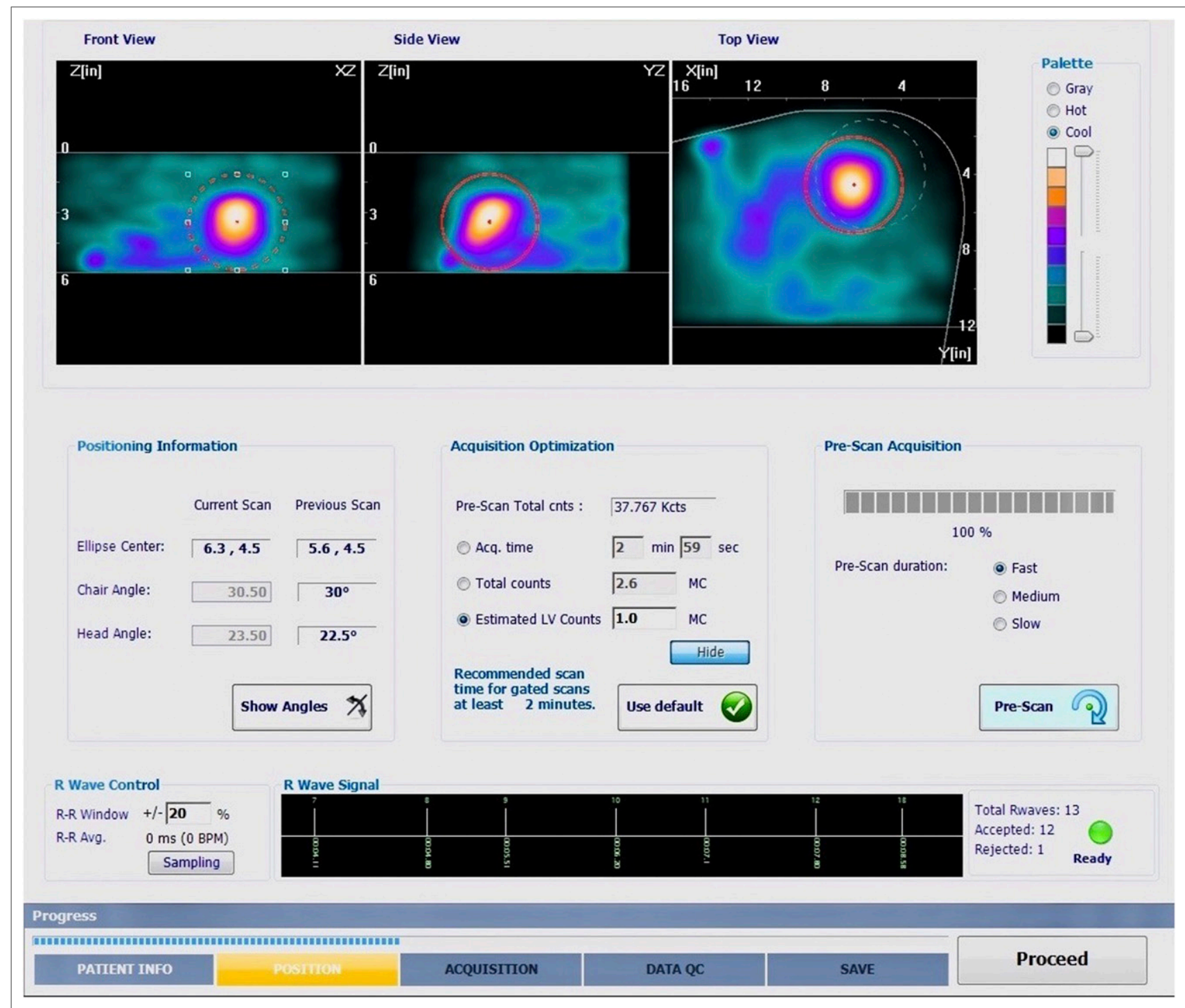

FIGURE 4. Prescan ensures that heart is accurately centered within FOV. Front and side views indicate superior and inferior limits. Dashed white circle in top view is D-SPECT indicator for lateral left side heart alignment; for appropriate imaging, heart must touch this circle, at minimum. Red circle needs to be adjusted to fit around myocardium on all 3 views for optimum count statistics.

A D-SPECT acquisition using technetium-labeled perfusion agents typically takes $2-8 \mathrm{~min}$ for standard doses according to the as-low-as-reasonably-achievable principle. Commonly used doses for the D-SPECT are shown in Table 2. Lower doses can be used but would require longer imaging times. Rest and stress images are acquired with 4lead electrocardiograms, and multiple acquisitions in the upright and the supine positions help to identify any breast or diaphragmatic attenuation artifacts. When using pharmacologic stress protocols while the patient is on the scanner, a separate 12-lead electrocardiogram may be required, as the camera is specifically designed to be used with 4 leads.

\section{Postprocessing}

The reconstruction algorithm on the D-SPECT is based on 3-dimensional maximum-likelihood expectation maxi- mization iterative reconstruction. This algorithm helps overcome the loss in spatial resolution caused by the larger collimator holes (3). Multiple processing platforms are available, similar to conventional SPECT systems. Processing accurately is more contingent on the initial setup and the prescan for the D-SPECT than for conventional processing systems. D-SPECT alignment is myocardium-specific because of the smaller detectors and abridged FOV. Therefore, alignment requires a more precise centering of the heart (4). Drawing accurate left ventricular regions of interest from the initial prescan, and at processing, is necessary for proper quality control of the images (Fig. 5) (4).

\section{Room Requirements}

The D-SPECT has a considerably smaller footprint than conventional SPECT systems. Depending on the system, 


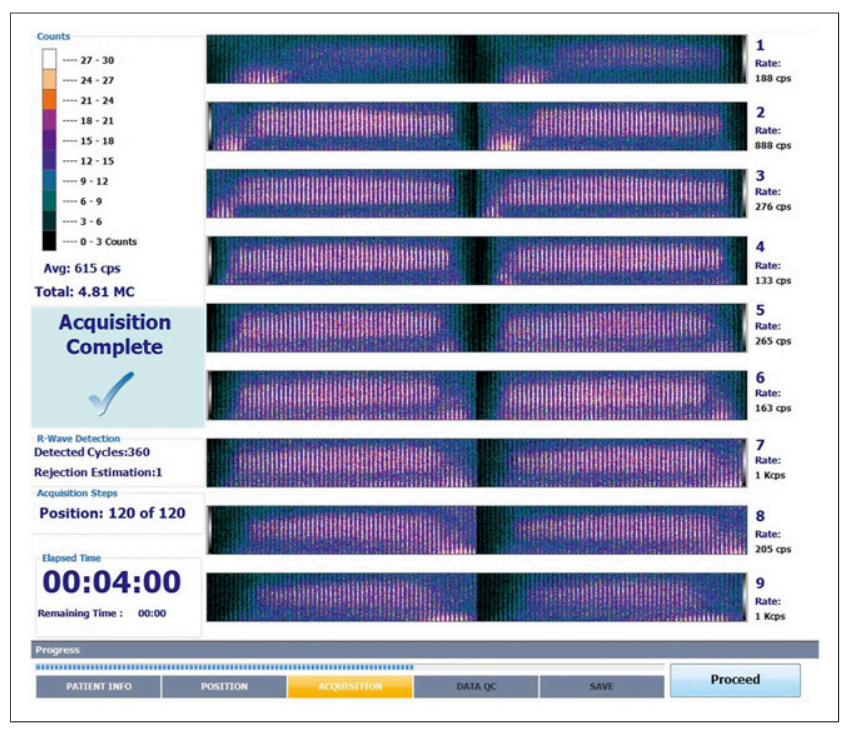

FIGURE 5. D-SPECT postscan. If prescan centering was accurate, each of 9 camera FOVs will be centered as in this image. Two separate vertical columns, left and right, represent first and second sweeps of detector. Scanning by the 9 detectors is based on regions of interest drawn by technologist, and then all detectors shift on linear motor and scan again. Afterward, these 2 sweeps are zipped together. Vertical lines are positions (equal to stops on traditional scanner), of which there are 60 for each detector per scan. Dark space above and below vertical lines indicates accurate prescan FOV alignment.

the D-SPECT fits in a room as small as $2.9 \times 3.4 \mathrm{~m}(9 \mathrm{ft} 5 \mathrm{in}$ $\times 11 \mathrm{ft})(15)$. In actual practice, for comfort and safety, the room should be closer to $3.0 \times 3.7 \mathrm{~m}(10 \times 12 \mathrm{ft})$ to allow for dynamic imaging, injection of pharmacologic agents, and additional personnel. This is still considerably less space than required for conventional nuclear medicine or SPECT/CT cameras, which typically require rooms of $4.6 \times$ $6.4 \mathrm{~m}(15 \times 21 \mathrm{ft})$ or larger.

\section{Limitations}

The main limitation of the D-SPECT system is the inability to correct for attenuation, creating challenges in the interpretation of some exams. Depending on body habitus, breast and diaphragmatic artifacts can limit the correct assessment of the anterolateral and inferolateral segments of the left ventricle. The possibility of acquiring images in different positions helps overcomes this issue. These artifacts may hamper the use of stress-only imaging.

The initial setup for subjects with a large body is also challenging. It is key to position the patient's heart in the FOV (displayed on the acquisition monitor) during the prescan step, but the heart sometimes will not fit into the FOV if excessive body tissue extends from the left side of the heart to the patient's exterior left side, which is in contact with the detector (Fig. 6).

Although rarely a problem, patients with severe cardiomegaly may pose issues to the imaging technologist if the

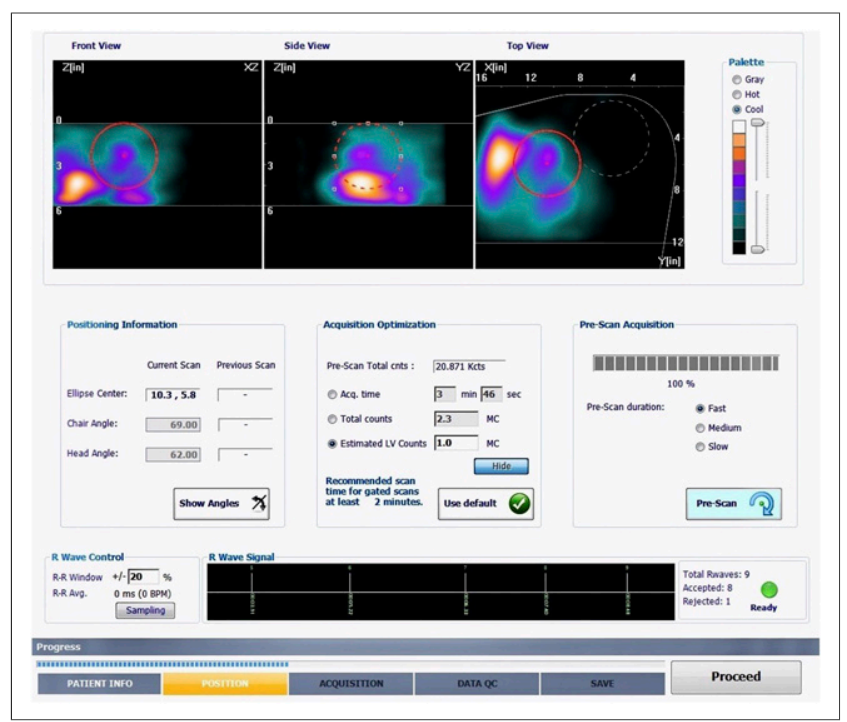

FIGURE 6. Example of poor positioning on D-SPECT prescan interface. Though frontal and side views are aligned within superior and inferior limits, top-view target area (dashed white circle) shows that heart is outside FOV. This can occur when patient is obese or when patient or camera is not adequately left-aligned.

heart is larger than the FOV. During processing, portions of the myocardium could be cut out, resulting in suboptimal diagnostic images (17).

In a hospital setting, one of the larger drawbacks may be the inability of the D-SPECT to handle immobile or uncooperative patients. The chair position is not adequate for patients who cannot sit upright on their own, patients with involuntary movements, and some patients with altered mental status.

\section{CONCLUSION}

D-SPECT technology has been shown to be a valid, safe, and accurate option for myocardial perfusion imaging studies, with benefits over SPECT imaging. Although CT attenuation technology, which is not available on the D-SPECT, increases the diagnostic value of conventional SPECT technology, the improved spatial resolution and sensitivity of the D-SPECT provides myocardial perfusion imaging studies of diagnostic quality. The proximity of the detector to the patient improves image quality while also reducing artifacts caused by patient movement. This close proximity also allows for a reduced dose or imaging time, both of which benefit the patient and decrease exposure to the technologist. Arguably, the most advantageous feature of the D-SPECT is the availability of upright imaging, which increases patient comfort. Superior image quality, low doses, and fast acquisition times, combined with optimal patient comfort, demonstrate that D-SPECT technology is advantageous both to the patient and to the nuclear medicine technologist acquiring the study.

\section{DISCLOSURE}

No potential conflict of interest relevant to this article was reported. 


\section{REFERENCES}

1. Benjamin EJ, Muntner P, Alonso A, et al. Heart disease and stroke statistics: 2019 update-a report from the American Heart Association. Circulation. 2019;139:e56e528.

2. Beller GA, Zaret BL. Contributions of nuclear cardiology to diagnosis and prognosis of patients with coronary artery disease. Circulation. 2000;101:1465-1478.

3. Agostini D, Marie P-Y, Ben-Haim S, et al. Performance of cardiac cadmiumzinc-telluride gamma camera imaging in coronary artery disease: a review from the cardiovascular committee of the European Association of Nuclear Medicine (EANM). Eur J Nucl Med Mol Imaging. 2016;43:2423-2432.

4. Dorbala S, Ananthasubramaniam K, Armstrong IS, et al. Single photon emission computed tomography (SPECT) myocardial perfusion imaging guidelines: instrumentation, acquisition, processing, and interpretation. J Nucl Cardiol. 2018;25:1784-1846.

5. Erlandsson K, Kacperski K, van Gramberg D, Hutton BF. Performance evaluation of D-SPECT: a novel SPECT system for nuclear cardiology. Phys Med Biol. 2009;54:2635-2649.

6. Gambhir SS, Berman DS, Ziffer J, et al. A novel high-sensitivity rapid-acquisition single-photon cardiac imaging camera. J Nucl Med.2009;50:635-643.

7. Alexiev D, Mo L, Prokopovich D, Smith ML, Matuchova M. Comparison of $\mathrm{LaBr} 3: \mathrm{Ce}$ and $\mathrm{LaCl} 3: \mathrm{Ce}$ with $\mathrm{NaI}(\mathrm{Tl})$ and cadmium zinc telluride (CZT) detectors. IEEE Trans Nucl Sci. 2008;55:1174-1177.

8. Burrell S, MacDonald A. Artifacts and pitfalls in myocardial perfusion imaging. J Nucl Med Technol. 2006;34:193-211.

9. Henzlova MJ, Duvall WL, Einstein AJ, Travin MI, Verberne HJ. ASNC imaging guidelines for SPECT nuclear cardiology procedures: stress, protocols, and tracers. J Nucl Cardiol. 2016;23:606-639.
10. Perrin M, Djaballah W, Moulin F, et al. Stress-first protocol for myocardial perfusion SPECT imaging with semiconductor cameras: high diagnostic performances with significant reduction in patient radiation doses. Eur J Nucl Med Mol Imaging. 2015;42:1004-1011.

11. Ben-Haim S, Almukhailed $\mathrm{O}$, Neill $\mathrm{J}$, et al. Clinical value of supine and upright myocardial perfusion imaging in obese patients using the D-SPECT camera. $J$ Nucl Cardiol. 2014;21:478-485.

12. Duvall WL, Sweeny JM, Croft LB, et al. Comparison of high efficiency CZT SPECT MPI to coronary angiography. $J$ Nucl Cardiol. 2011;18:595-604.

13. Duvall WL, Sweeny JM, Croft LB, Ginsberg E, Guma KA, Henzlova MJ. Reduced stress dose with rapid acquisition CZT SPECT MPI in a non-obese clinical population: comparison to coronary angiography. J Nucl Cardiol. 2012;19:1927.

14. Botvinick EH, Zhu YY, O'Connell WJ, Dae MW. A quantitative assessment of patient motion and its effect on myocardial perfusion SPECT images. $J$ Nucl Med. 1993;34:303-310.

15. Case JA, Bateman TM. Taking the perfect nuclear image: quality control, acquisition, and processing techniques for cardiac SPECT, PET, and hybrid imaging. J Nucl Cardiol. 2013;20:891-907.

16. Allie R, Hutton BF, Prvulovich E, Bomanji J, Michopoulou S, Ben-Haim S. Pitfalls and artifacts using the D-SPECT dedicated cardiac camera. J Nucl Cardiol. 2016;23:301-310.

17. Rosenthal MS, Cullom J, Hawkins W, Moore SC, Tsui BM, Yester M. Quantitative SPECT imaging: a review and recommendations by the Focus Committee of the Society of Nuclear Medicine Computer and Instrumentation Council. J Nucl Med. 1995;36:1489-1513. 